\title{
Reconhecimento de fala em crianças usuárias de implante coclear utilizando dois diferentes processadores de fala
}

\section{Speech recognition in children with cochlear implants using two different speech processors}

\author{
Fabiana Danieli', Maria Cecília Bevilacqua²
}

\begin{abstract}
RESUMO
Objetivo: Estudar comparativamente a habilidade de reconhecimento de fala no silêncio e na presença de ruído competitivo em crianças usuárias de implante coclear utilizando dois diferentes processadores de fala. Métodos: Foram avaliadas 26 crianças usuárias do implante coclear Nucleus 24M/24K, da Cochlear Corporation $®$, divididas em dois grupos de acordo com o processador de fala utilizado. O Grupo 1 foi composto por 16 crianças que faziam uso do processador de fala Sprint e o Grupo 2 foi composto por 10 crianças que faziam uso do processador de fala Freedom. Foi aplicado o Hearing in Noise Test - versão em Português/ Brasil, em campo livre, na condição de silêncio e na presença de ruído competitivo. Resultados: O desempenho do Grupo 2 (Freedom) foi superior ao desempenho do Grupo 1 (Sprint) em todas as condições de avaliação, sendo evidenciada diferença entre eles na condição de silêncio. Conclusão: $O$ processador de fala apresentou influência significativa na percepção de fala de crianças usuárias dos implantes cocleares Nucleus $24 \mathrm{~K}$ e $24 \mathrm{M}$. As características de pré-processamento do som presentes no processador de fala Freedom podem ter contribuído para o melhor desempenho do Grupo 2 nos testes de percepção de fala realizados. Novos estudos são necessários para complementação destes achados.
\end{abstract}

Descritores: Implante coclear; Criança; Audição; Ruído; Perda auditiva

\begin{abstract}
Purpose: To study comparatively the speech recognition skills in quiet and noise background situation in children with cochlear implants using two different speech processors. Methods: It was evaluated 26 children with cochlear implant device Nucleus $24 \mathrm{M}$ or $24 \mathrm{~K}$, Cochlear Corporation, divided in two groups according to the speech processor used. The Group 1 consisted of 16 children who used the Sprint processor and the Group 2 consisted of 10 children who used the Freedom processor. The Hearing in Noise Test - Portuguese version of Brazil was applied in the sound field in silence and in the presence of noise background situation. Results: The speech recognition performance of Group 2 (Freedom) was higher than Group 1 (Sprint) in all evaluation situations, with significant difference between groups only on quiet situation. Conclusion: The speech processor showed significant influence on speech perception in children users of Nucleus $24 \mathrm{~K}$ and $24 \mathrm{M}$ cochlear implants. The components of the signal processing available on the Nucleus Freedom processor may have contributed to the better speech recognition performance of Group 2. Further research is needed to complement these findings.
\end{abstract}

Keywords: Cochlear implantation; Child; Hearing; Noise; Hearing loss

Trabalho realizado como conclusão do Curso de Mestrado pelo Programa de Pós-Graduação Interunidades em Bioengenharia, Escola de Engenharia de São Carlos/ Instituto de Química de São Carlos/ Faculdade de Medicina de Ribeirão Preto - EESC/IQSC/FMRP-USP - São Carlos (SP), Brasil.

(1) Programa de Pós-graduação (Mestrado) Interunidades em Bioengenharia, Escola de Engenharia de São Carlos/ Instituto de Química de São Carlos/ Faculdade de Medicina de Ribeirão Preto - EESC/IQSC/FMRP-USP - São Carlos (SP), Brasil.

(2) Departamento de Fonoaudiologia, Faculdade de Odontologia de Bauru e Hospital de Reabilitação de Anomalias Craniofaciais, Universidade de São Paulo USP - Bauru (SP), Brasil.

Contribuição dos autores: Os autores $(F D, M C B)$ foram responsáveis pela elaboração da pesquisa e do artigo científico.

Conflito de interesses: Não

Endereço para correspondência: Fabiana Danieli. Al. Campinas,1183/33, Jardim Paulista, São Paulo (SP), Brasil, CEP: 01404-001. E-mail: fabianadanieli@hotmail.com

Recebido em: 21/11/2011; Aceito em: 4/2/2013 


\section{INTRODUÇÃO}

A indicação do implante coclear (IC) para crianças com deficiência auditiva severa e/ou profunda tem aumentado drasticamente em todo o mundo. Este crescente aumento tem impulsionado o desenvolvimento de novas tecnologias que visam melhorar cada vez mais o desempenho dos usuários deste dispositivo, principalmente em situações de difícil compreensão, como na presença de ruído competitivo.

A percepção da fala em condições desfavoráveis, como na presença de reverberação e ruídos competitivos ${ }^{(1)}$ é um dos temas mais preocupantes para usuários de implante coclear. Estudo realizado demonstrou uma queda significativa no desempenho de percepção de fala de implantados na presença de ruído competitivo ${ }^{(2)}$, sendo esta a queixa mais frequente entre eles ${ }^{(3)}$. De acordo com pesquisador ${ }^{(4)}$, a integridade do processador de fala e de seus algoritmos constitui um fator determinante para a habilidade do usuário em compreender a fala e também separar o sinal almejado de todos os outros sinais, incluindo o ruído. Pesquisas recentes demonstraram benefícios na percepção de fala dos usuários de implante coclear proporcionados por inovações tecnológicas dos dispositivos, incluindo processador de fala e demais componentes responsáveis pelo algoritmo processamento do som ${ }^{(1,5,6)}$.

O processador de fala está presente em todos os sistemas de implante coclear e corresponde ao principal componente externo do dispositivo. Nele são selecionados todos os parâmetros responsáveis pela determinação do modo pelo qual o sistema de implante coclear irá transformar o sinal acústico da fala em impulsos elétricos, responsáveis por estimular as fibras nervosas remanescentes na cóclea. Estes parâmetros incluem desde microfone e suas características de pré-processamento do som, até as estratégias de codificação da fala, que correspondem a um dos principais parâmetros do sistema a ser selecionado no software de programação. As estratégias de codificação definem o modo como o processador irá analisar e codificar as informações importantes da fala, bem como transformar as informações codificadas em pulsos elétricos, determinando, ainda, como eles serão entregues aos eletrodos inseridos na cóclea.

O rápido avanço da tecnologia tem promovido o desenvolvimento de processadores de fala cada vez mais sofisticados, que associados a novas estratégias de processamento do som e softwares de programação mais completos, visam melhorar ainda mais a qualidade dos sons de fala e eliminar o ruído competitivo. Entretanto, fica a dúvida se esses novos processadores poderiam realmente promover melhores desempenhos também para usuários de prévias gerações de implantes cocleares.

Dessa forma, este estudo teve como objetivo estudar comparativamente a habilidade de reconhecimento de fala no silêncio e na presença de ruído competitivo em crianças usuárias de implante coclear utilizando dois diferentes processadores de fala.

\section{MÉTODOS}

Este estudo foi desenvolvido no Centro de Pesquisas Audiológicas do Hospital de Reabilitação de Anomalias Craniofaciais (HRAC/USP), no setor de Implante Coclear. Foi aprovado pelo Comitê de Ética em Pesquisa da Faculdade de Odontologia de Bauru da Universidade de São Paulo (FOB/ USP), sob o parecer $n^{\circ}$ 093/2009. Foram avaliadas 26 crianças de ambos os gêneros, usuárias do sistema de implante coclear Nucleus ${ }^{\circledR 24}$, da Cochlear Corporation $®$, divididas em dois grupos de acordo com o processador de fala utilizado, denominados Grupo 1 e Grupo 2.

O Grupo 1 foi composto por 16 crianças usuárias do implante coclear Nucleus CI24M ou CI24R(ST), que faziam uso do processador de fala Sprint. O Grupo 2 foi composto por 10 crianças usuárias do implante coclear Nucleus CI24M ou CI24R(ST), que faziam uso do processador de fala Freedom.

Foram obtidos os dados referentes à distribuição dos grupos estudados quanto à idade na época do experimento (IDD), tempo de privação sensorial auditiva (TPRI) e tempo de uso do dispositivo de implante coclear (TUSO) (Tabela 1).

Tabela 1. Distribuição dos grupos estudados quanto à idade na época do experimento, tempo de privação sensorial auditiva e tempo de uso do dispositivo de implante coclear

\begin{tabular}{|c|c|c|c|c|c|}
\hline & $\begin{array}{c}\text { Sujeitos } \\
(n)\end{array}$ & & $\begin{array}{c}\text { IDD } \\
\text { (meses) }\end{array}$ & $\begin{array}{c}\text { TPRI } \\
\text { (meses) }\end{array}$ & $\begin{array}{c}\text { TUSO } \\
\text { (meses) }\end{array}$ \\
\hline \multirow{5}{*}{ Grupo 1 (Sprint) } & \multirow{5}{*}{16} & Média & 98,19 & 25,23 & 71,88 \\
\hline & & DP & 26,48 & 10,28 & 18,15 \\
\hline & & Mediana & 92,50 & 23,00 & 67,00 \\
\hline & & Mínimo & 62,00 & 13,00 & 46,00 \\
\hline & & Máximo & 144,00 & 47,00 & 98,00 \\
\hline \multirow{5}{*}{ Grupo 2 (Freedom) } & \multirow{5}{*}{10} & Média & 118,30 & 27,90 & 87,80 \\
\hline & & $\mathrm{DP}$ & 33,70 & 11,00 & 26,45 \\
\hline & & Mediana & 118,50 & 23,50 & 91,50 \\
\hline & & Mínimo & 74,00 & 17,00 & 52,00 \\
\hline & & Máximo & 186,00 & 50,00 & 137,00 \\
\hline
\end{tabular}

Legenda: IDD = idade na época do experimento;TPRI = tempo de privação sensorial auditiva; TUSO = tempo de uso do dispositivo de implante coclear; DP = desvio-padrão 
Todas as crianças avaliadas apresentavam perda auditiva neurossensorial de grau severo e/ou profundo ou profundo bilateral, de origem pré-lingual, com cirurgia realizada no HRAC/ USP até 4 anos e 2 meses de idade. O tempo mínimo de uso do implante coclear considerado para a seleção das crianças foi de 3 anos e para o processador de fala foi de 5 meses. O tempo de experiência com o processador de fala Freedom (Grupo 2) variou de 5 meses a 3 anos. Todas as crianças apresentaram inserção total dos eletrodos na cóclea.

As crianças avaliadas apresentavam a habilidade auditiva de reconhecimento de fala em conjunto aberto ("open-set"), de acordo com as categorias de audição( ${ }^{(7)}$ e linguagem oral fluente, de acordo com categorias de linguagem ${ }^{(8)}$. Ainda, todas as crianças apresentavam características homogêneas, mesmo nível socioeconômico e estavam regularmente matriculadas e acompanhadas pelo programa de Implante Coclear do HRAC/USP.

Foi realizada a distribuição dos grupos estudados quanto à etiologia da deficiência auditiva (Tabela 2).

Tabela 2. Distribuição dos grupos estudados quanto à etiologia da deficiência auditiva

\begin{tabular}{lccccccc}
\hline \multirow{2}{*}{ Etiologia } & \multicolumn{3}{c}{$\begin{array}{c}\text { Frequências absolutas }(\mathrm{n}) \\
\text { e porcentagens (\%) }\end{array}$} & \multirow{2}{*}{ Total } \\
\cline { 2 - 5 } & \multicolumn{2}{c}{ Grupo 1 } & \multicolumn{2}{c}{ Grupo 2 } & & \\
\hline Genética & 0 & 0 & 3 & 18,75 & 3 & 11,54 \\
Idiopática & 6 & 60,00 & 12 & 75,00 & 18 & 69,23 \\
Meningite & 1 & 10,00 & 0 & 0 & 1 & 3,85 \\
Rubéola & 3 & 30,00 & 1 & 6,25 & 4 & 15,38 \\
\hline Total & 10 & 100,00 & 16 & 100,00 & 26 & 100,00 \\
\hline
\end{tabular}

Os principais parâmetros de programação selecionados nos Grupos 1 e 2 também foram obtidos (Tabela 3).

Tabela 3. Principais parâmetros de programação selecionados nos Grupos 1 e 2

\begin{tabular}{lcc}
\hline Parâmetros & Grupo 1 & Grupo 2 \\
\hline Eletrodos ativos & 22 & 22 \\
Modo de estimulação & MP1+2 & MP1+2 \\
Estratégia de codificação da fala & ACE & ACE \\
Número de máximas & 12 & 12 \\
Velocidade de estimulação/canal & 1200 PPS & $1200 \mathrm{PPS}$ \\
Distribuição de frequências & $188-7938 \mathrm{~Hz}$ & $188-7938 \mathrm{~Hz}$ \\
\hline
\end{tabular}

Todos os sujeitos do Grupo 2, usuários do processador de fala Freedom, faziam uso de Adaptative Dynamic Range Optimization (ADRO) e de uma Instantaneous Input Dynamic Range (IIDR) mais ampla, de $40 \mathrm{~dB}$.

Foi realizada avaliação do componente externo (processador de fala, antena transmissora, microfone e cabos) por meio de inspeção manual e do componente interno, por meio dos sistemas de telemetria: telemetria de impedância, telemetria de compliância e telemetria de respostas neurais (NRT). Estes procedimentos foram realizados com o objetivo de descartar qualquer problema com o dispositivo.

Os participantes da pesquisa foram avaliados durante o retorno ao Centro de Pesquisas Audiológicas para acompanhamento (etapa pós-cirúrgica). A avaliação da percepção de fala foi realizada com o processador de fala programado no mapa realizado desde o último retorno, mais utilizado pela criança.

Os retornos para acompanhamento na etapa pós-cirúrgica são realizados a cada três meses no primeiro ano de uso do dispositivo, a cada seis meses no segundo ano, e tornam-se mais espaçados conforme a experiência com o uso do IC vai se tornando maior. Isto ocorre, entre outros fatores, porque os limiares para a estimulação elétrica variam ao longo do tempo, principalmente nos períodos iniciais de programação, mas tendem a se estabilizar conforme o indivíduo vai adquirindo experiência com o dispositivo. Assim, os mapas contendo os limiares de estimulação elétrica, criados no processador de fala, são ajustados por meio de um processo gradual de ajuste fino com o decorrer do tempo. Para avaliar a percepção de fala, as crianças foram instruídas a utilizar somente o sistema de implante coclear, descartando o uso do AASI contralateral.

O protocolo de avaliação da percepção de fala foi composto pela versão adaptada para o Português/Brasil do Hearing in Noisy Test $(\mathrm{HINT})^{(9)}$. O HINT corresponde a um teste de reconhecimento de fala com e sem a presença de ruído competitivo. É um teste adaptativo, onde a intensidade das sentenças varia de acordo com a resposta do sujeito. Ele engloba 240 sentenças distribuídas em 12 listas com 20 sentenças cada, foneticamente balanceadas, que podem ser aplicadas com a utilização de fones de ouvido ou em campo livre. Seu resultado é expresso em Limiar de Recepção de Sentenças (LRS) e quanto menor o valor do LRS melhor o desempenho do sujeito.

O HINT foi desenvolvido com o intuito de aprimorar a qualidade da tecnologia dos dispositivos auxiliares de audição, como os aparelhos auditivos e implantes cocleares ${ }^{(10)}$. Sua aplicação para avaliar o desempenho de usuários de implante coclear tem sido cada vez maior por ser um teste eficiente, rápido, de fácil aplicação e que fornece medidas confiáveis e resultados passíveis de repetições. Além disso, ele ainda permite uma comparação confiável dos resultados obtidos com diversos outros países, já que foi normatizado para diferentes idiomas.

O HINT permite avaliar a capacidade funcional auditiva, ou seja, determinar o quão hábil uma pessoa é para ouvir e entender a fala principalmente em ambientes ruidosos. Tendo em vista o impacto negativo do ruído na comunicação, os testes de percepção de fala na presença de ruído competitivo refletem de maneira mais eficiente as condições de escuta do dia-a-dia, possibilitando uma avaliação detalhada da capacidade de comunicação das pessoas ${ }^{(11)}$.

Neste estudo, foi aplicada uma lista com 20 sentenças para cada condição de teste, escolhida aleatoriamente pelo próprio 
software do HINT, em campo livre. Foi aplicado na condição de silêncio $(S)$ e na presença de ruído competitivo ( $S / R$ $\left.180^{\circ}\right)$. Na condição de silêncio as sentenças foram aplicadas sem a presença de ruído competitivo, na posição frontal $\left(0^{\circ}\right.$ azimute), por meio de caixa acústica posicionada a um metro de distância da criança (Figura 1A). Na presença de ruído competitivo, as sentenças foram aplicadas na posição frontal $\left(0^{\circ}\right.$ azimute) e o ruído competitivo foi posicionado atrás do paciente ( $180^{\circ}$ azimute). As sentenças e o ruído competitivo foram aplicados por meio de caixas acústicas localizadas a um metro de distancia do sujeito, nas posições $0^{\circ}$ e $180^{\circ}$ azimute (Figura 1B).

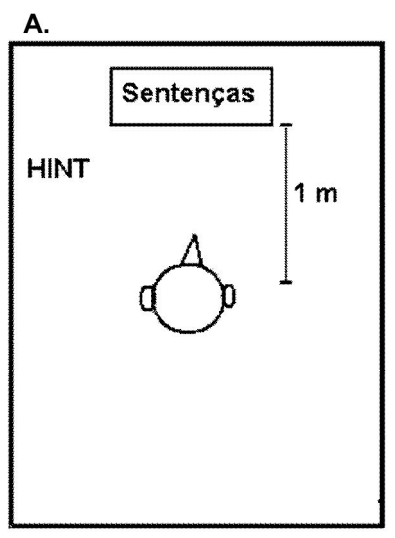

B.

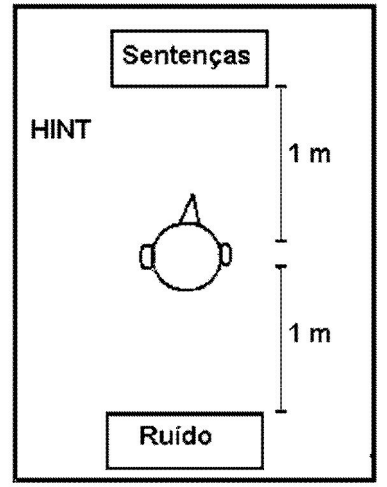

Figura 1. A. Posição da cabeça do paciente em relação à caixa acústica para a aplicação do HINT na condição silêncio (S). B. Posição da cabeça do paciente em relação às caixas acústicas para a aplicação do HINT na condição silêncio (S)

Optou-se pelo emprego de métodos de análise descritiva, visando um estudo exploratório exaustivo e de consistência dos dados. Então, foi realizada uma comparação dos grupos experimentais em relação às variáveis dependentes ( $\mathrm{S}$ e $\mathrm{S} / \mathrm{R} 180^{\circ}$ ), por meio do teste t. O nível de significância adotado para todos os testes estatísticos foi de $5 \%$.

\section{RESULTADOS}

Foi realizada a comparação entre os Grupos 1 e 2 com relação à idade (IDD), tempo de privação sensorial auditiva (TPRI) e tempo de uso do sistema de implante coclear (TUSO) (Figura 2). A distribuição dos grupos apresentou-se similar para as três variáveis estudadas, com valores médios discretamente mais elevados para o Grupo 2.

Diversos estudos demonstraram influência significativa destas variáveis no desempenho de percepção de fala de crianças usuárias de implante coclear ${ }^{(12,13)}$. Dessa forma, houve grande preocupação em seu controle para a avaliação dos grupos.

Observa-se que o Grupo 2 apresentou em média e mediana, maiores idades que o grupo 1 , bem como maior variabilidade na idade ( $\mathrm{DP}=33,70$ meses) (Figura 1). Entretanto, a comparação das médias de idade nos dois grupos pela aplicação do teste t bilateral não indicou, ao nível de até 5\% de significância,

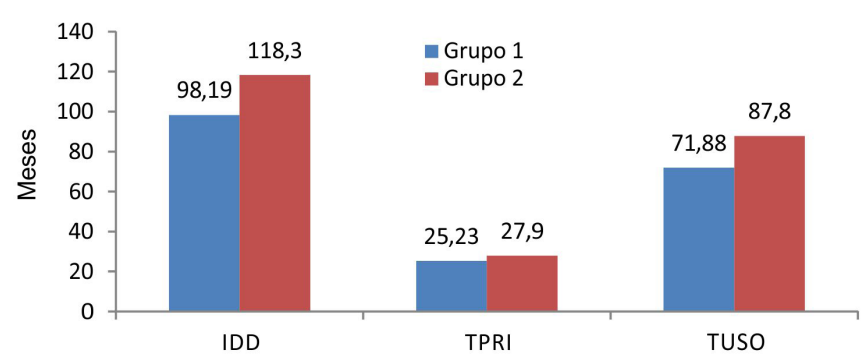

Legenda: IDD = idade na época do experimento; TPRI = tempo de privação sensorial auditiva; TUSO = tempo de uso do dispositivo de implante coclear

Figura 2. Comparação da idade, tempo de privação sensorial auditiva e tempo de uso do Implante Coclear entre os grupos

diferenças significativas entre as idades nos dois grupos $(\mathrm{p}=0,130 ; \mathrm{p}>0,05)$.

Os Grupos 1 e 2 apresentaram, em média e medianamente, tempo de privação sensorial auditiva e variabilidades aproximadamente iguais. Os resultados do teste $t$ indicaram não existir diferenças significativas entre os grupos para o tempo de privação sensorial auditivas das crianças ( $\mathrm{p}=0,605$; $\mathrm{p}>0,05$ ).

Em relação ao tempo de uso do dispositivo, o Grupo 2 apresentou maiores médias, medianas e também variabilidades no tempo de uso do que o grupo 1. Estes resultados provavelmente ocorreram em função da distribuição da idade das crianças neste grupo. Observou-se que o Grupo 2 apresentou, em média e medianamente, maiores idades que o Grupo 1, bem como maior variabilidade na idade.

A comparação das médias do tempo de uso do IC nos dois grupos, pela aplicação de um teste t bilateral, indicou não existir diferenças significativas entre os grupos, ao nível de até 5\% de significância $(\mathrm{p}=0,116 ; \mathrm{p}>0,05)$.

Foi descrito o desempenho dos dois grupos estudados para o HINT na condição de Silêncio (S) (Figura 3). O Grupo 2 apresentou um valor médio de LRS mais baixo que o Grupo 1 , demonstrando melhor desempenho nesta condição de teste. $\mathrm{O}$ teste $\mathrm{t}$ revelou que esta diferença foi significativa $(\mathrm{p}=0,053$; $p>0,05$ ), embora o valor de $p$ tenha se encontrado muito próximo do nível de significância de referência.

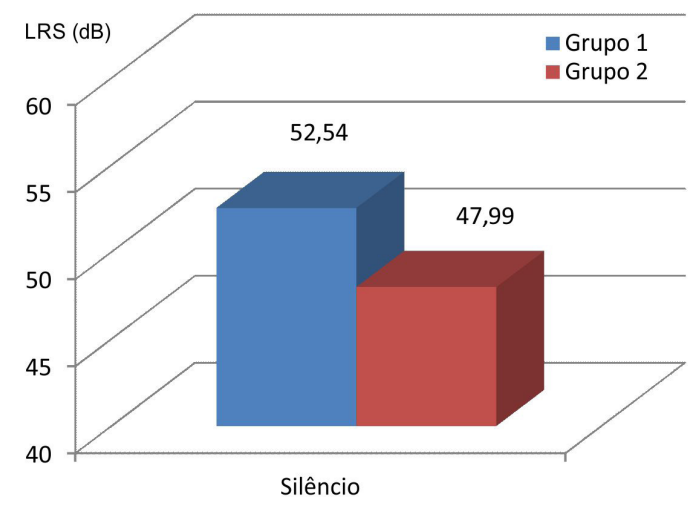

Figura 3. Valores médios do Limiar de Recepção de Sentenças (LRS) obtidos pelos Grupos 1 e 2 para o HINT na condição de silêncio 
O desempenho dos dois grupos estudados para o HINT na condição de ruído competitivo (S/R $180^{\circ}$ ) também foi observado (Figura 4). O Grupo 2 apresentou um valor de LRS mais baixo que o Grupo 1, demonstrando desempenho médio superior deste grupo na presença de ruído competitivo. Entretanto, embora o Grupo 2 tenha apresentado um desempenho médio superior para a condição de ruído competitivo, o teste t demonstrou não haver diferença significativa entre o desempenho dos grupos para esta condição $(\mathrm{p}=0,016 ; \mathrm{p}>0,05)$.

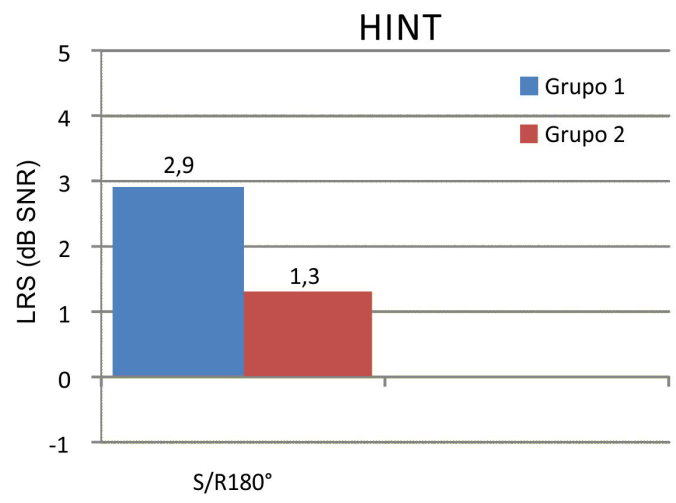

Figura 4. Comparação das médias dos resultados obtidos pelos Grupos 1 e 2 para o HINT na condição S/R180

Foi realizada a distribuição dos resultados obtidos pelos Grupos 1 e 2 para a condição de ruído competitivo (S/R 180) (Figura 5). Observou-se a presença de um ponto atípico na distribuição das respostas do Grupo 2, tornando os resultados médios e medianos deste grupo bastante discrepantes.

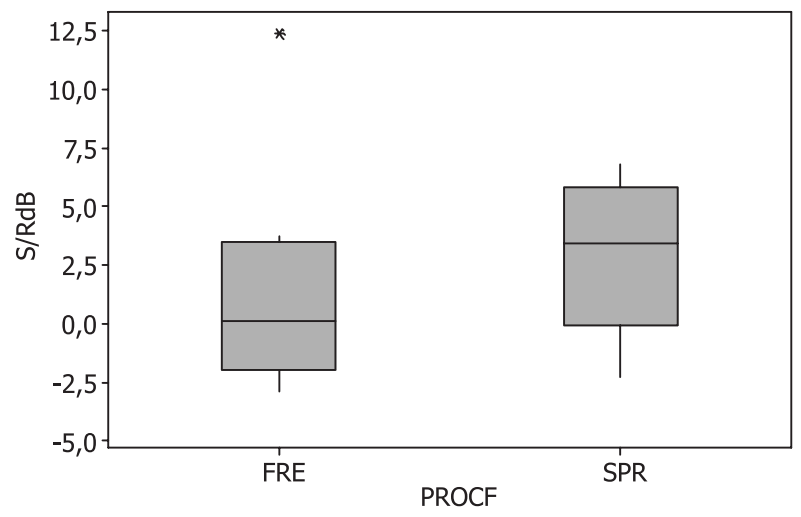

Figura 5. Resultados obtidos para os Grupos 1 e 2 para a condição de ruído competitivo (S/R $\left.180^{\circ}\right)$

O sujeito 7, referente ao ponto atípico no Grupo 2, apresentou uma pontuação relativamente alta comparada às demais pontuações obtidas por este grupo $(12,4 \mathrm{~dB}$ SNR comparado à média de resultados deste grupo, que está por volta de $1,30 \mathrm{~dB}$ SNR). O mesmo não ocorreu na condição de silêncio, em que o sujeito apresentou o limiar de reconhecimento de sentenças HINT em 55,5 dB, sendo portanto, simétrico à distribuição dos resultados obtidos pelos demais sujeitos do grupo.

Para o HINT na presença de ruído competitivo (S/R180º), notou-se que, com ou sem a inclusão do ponto atípico no Grupo 2 , houve um desempenho superior deste grupo em relação ao grupo 1. Entretanto, somente com a exclusão do ponto atípico, estes resultados evidenciaram diferenças significativas entre os grupos.

Retirando-se o ponto atípico do Grupo 2, os valores para a condição $\mathrm{S} / \mathrm{R} 180^{\circ}$ se mantiveram, em média e medianamente, maiores no Grupo 1 (2,975 dB SNR e 3,45 dB SNR respectivamente para o Grupo 1, comparados a 0,067 dB SNR e -0,30 dB SNR respectivamente para o Grupo 2), evidenciando melhor desempenho do Grupo 2. A diferença dos valores obtidos para os grupos torna-se mais discrepante quando o ponto atípico do grupo é retirado do Grupo 2. Ao comparar os valores médios obtidos pelos grupos, o teste t bilateral rejeitou, ao nível de $5 \%$ de significância, a igualdade entre as médias dos valores da variável $\mathrm{S} / \mathrm{R}$ nos grupos 1 e $2(\mathrm{p}=0,016 ; \mathrm{p}<0,05)$.

\section{DISCUSSÃO}

Este estudo buscou investigar comparativamente a habilidade de reconhecimento de fala no silêncio e na presença de ruído competitivo em crianças usuárias de implante coclear e dois diferentes tipos de processadores de fala. Dessa forma, o objetivo foi verificar se diferentes processadores poderiam influenciar na habilidade de reconhecimento de fala no silêncio e na presença de ruído competitivo.

Verificou-se que as crianças usuárias do processador de fala Freedom apresentaram desempenho superior ao das crianças que faziam uso do processador de fala Sprint em todas as condições de avaliação. Entretanto, foi evidenciada diferença entre os grupos apenas para a percepção de fala no silêncio. $\mathrm{O}$ desempenho superior do processador de fala Freedom nos testes de percepção de fala em relação aos prévios processadores (Sprint, Esprit e Esprit 3G) para usuários de Nucleus 24M ou 24K foi relatado na literatura ${ }^{(14-16)}$.

Os sujeitos deste estudo apresentaram uma média de superioridade no desempenho com o processador de fala Freedom para reconhecimento de fala na presença de ruído competitivo de 1,68 dB SNR, comparado ao Sprint. Este resultado corrobora o estudo ${ }^{(14)}$ que apresentou uma média de melhora no desempenho dos sujeitos para reconhecimento de fala na presença de ruído competitivo com o processador de fala Freedom por volta de 1,8 dB SNR, em relação aos processadores Sprint, Esprit $3 G$ ou Esprit. Tais achados indicam a relevância na realização de estudos que investiguem os resultados dos diferentes modelos de processadores de fala.

Sabe-se que outras variáveis influenciam significativamente os resultados de percepção de fala de usuários de implante coclear, como tempo de privação sensorial auditiva, tempo de uso do IC e idade na percepção de fala ${ }^{(12,13,17)}$. Os resultados demonstraram que estas variáveis se mantiveram controladas entre os grupos avaliados neste estudo, descartando sua possível influência no desempenho dos grupos. 
Em relação à comparação da idade das crianças na época do experimento, observou-se uma pequena assimetria à direita, em direção aos maiores valores de idade, nas distribuições de ambos os grupos. Esta variabilidade provavelmente ocorreu em função da extensa distribuição da faixa etária avaliada no estudo, que foi de 5 anos e 2 meses a 15 anos e 6 meses. Não foi observada a presença de pontos atípicos, ou seja, crianças com idades muito discrepantes da principal massa de dados.

Considerando que o tempo de privação sensorial auditiva se apresentou simétrico e aproximadamente igual entre os grupos, é esperado que o tempo de uso do IC dos sujeitos do Grupo 2 seja maior, assim como a variabilidade encontrada. Também não foram observados pontos atípicos, ou seja, crianças com tempo de uso do IC muito discrepantes da principal distribuição dos dados.

A pontuação notavelmente alta do sujeito 7 , demonstrada pela presença do ponto atípico na distribuição dos resultados do Grupo 2 para a situação S/R $180^{\circ}$, evidenciou um pior desempenho deste indivíduo para a percepção de fala na presença de ruído competitivo. Esta situação foi considerada um fato isolado e deve ser cuidadosamente investigada se ocorrer em um ambiente clínico. Visto que houve uma grande preocupação em se estabelecer um controle das demais variáveis que poderiam interferir nos resultados do estudo, este fato poderia ser atribuído à habilidade de processamento auditivo deste sujeito, mais precisamente a habilidade de figura-fundo auditiva.

Em relação às características tecnológicas e de processamento do som nos processadores de fala estudados, foram selecionados os mesmos parâmetros principais de programação para ambos os grupos, demonstrados na Tabela 3. Embora tenha sido determinada a mesma estratégia de processamento do som para os dois grupos, tais processadores apresentam algumas características que se diferem em termos de pré-processamento do sinal sonoro. Dessa forma, estes algoritmos parecem ter influenciado significativamente no desempenho superior das crianças usuárias do processador de fala Freedom demonstrados neste estudo.

Estudo $^{(14)}$ demonstrou melhores resultados de reconhecimento de fala no silêncio para sujeitos que faziam uso do processador de fala Freedom com a ativação da Adaptative Dynamic Range Optimization (ADRO), comparado ao uso do Freedom sem a ativação da ADRO ou a outros processadores de fala como o Esprit, Sprit $3 G$ e Sprint. A ADRO ajusta dinamicamente o ganho de cada canal de estimulação de acordo com o nível do sinal de entrada deste canal. Este algoritmo otimiza o ganho de cada canal, mantendo a saída dentro de uma área dinâmica confortável ao paciente. Os canais que representam sons de baixa intensidade são reforçados, enquanto os sons de alta intensidade são atenuados. Todas as crianças usuárias do processador de fala Freedom faziam uso de ADRO, o que nos leva a pensar que este algoritmo pode ter influenciado significativamente no desempenho superior do Grupo 2 demonstrado neste estudo.
Existe um consenso em relação aos melhores resultados de percepção de fala no silêncio com a utilização do ADRO, entretanto, na presença de ruído competitivo as opiniões são bastante controversas. Estudo relatou a influência significativa da ADRO na percepção da fala na presença de ruído competiti$\mathrm{vo}^{(15)}$. Em contrapartida, outro estudo referiu que este algoritmo não tem efeito quando a fala está competindo com o ruído ${ }^{(18)}$.

O mesmo ocorre para a Instantaneous Input Dynamic Range (IIDR). Estudo ${ }^{(19)}$ demonstrou que usuários do processador de fala Freedom utilizando uma IIDR mais ampla (40 dB) apresentaram melhores resultados de percepção de fala no silêncio, principalmente em intensidades mais baixas, quando comparados ao uso do Freedom com IIDR de $30 \mathrm{~dB}$ ou aos demais processadores Sprint ou Esprit, com IIDR de 30 dB. A IIDR corresponde ao intervalo de intensidades acústicas selecionados para o mapeamento da área dinâmica de estimulação elétrica do usuário de IC. Todas as crianças usuárias do processador de fala Freedom faziam uso de uma IIDR mais ampla (40 dB), comparada à utilizada pelas crianças usuárias do processador de fala Sprint ( $30 \mathrm{~dB}$ ). Contudo, novos estudos são necessários para investigar com precisão a influência que cada componente do pré-processamento do som exerce na habilidade de percepção de fala do usuário.

Estudos relacionados aos algoritmos de processamento do som, presentes e ajustados ao nível do processador de fala, trazem uma importante contribuição para a seleção dos parâmetros na programação, visando otimizar a percepção de fala do usuário. Além disso, como descrito anteriormente, com o rápido avanço da tecnologia e o desenvolvimento de novos processadores de fala cada vez mais sofisticados, fica a dúvida se estes novos processadores poderiam promover um melhor desempenho também para usuários de prévias gerações de implantes cocleares. Desta forma, esta informação pode auxiliar de forma significativa na escolha de um novo processador de fala, tendo em vista o seu tempo de vida útil e demais problemas que acarretam na necessidade de sua reposição, bem como nortear políticas públicas e privadas relacionadas à troca dos processadores de fala.

Ainda, o presente estudo também traz importantes contribuições no que diz respeito ao aprimoramento das medidas dos resultados da percepção de fala em crianças usuárias de implante coclear no Brasil. A utilização do HINT torna possível a comparação dos resultados obtidos com diversos outros países, já que permite uma comparação dos resultados da audição funcional em diferentes idiomas.

\section{CONCLUSÃO}

O processador de fala apresenta influência significativa na percepção de fala de crianças usuárias dos implantes cocleares Nucleus $24 \mathrm{~K}$ e $24 \mathrm{M}$ com os processadores de fala Sprint e Freedom. Os algoritmos de pré-processamento do som presentes no processador de fala Freedom podem ter contribuído para o melhor desempenho do Grupo 2 nos testes realizados. 
Contudo, novos estudos são necessários para investigar com precisão a influência dos componentes dos processadores de fala, referentes ao pré-processamento e processamento do som, na habilidade de percepção de fala do usuário.

O HINT se mostrou um instrumento eficaz na avaliação de reconhecimento de fala no silêncio e na presença de ruído competitivo em crianças usuárias de implante coclear. Assim, os resultados encontrados neste estudo poderão ser utilizados comparativamente com os de outros países e em pesquisas futuras no Brasil.

\section{REFERÊNCIAS}

1. Frederigue NB, Bevilacqua MC. Otimização da percepção da fala em deficientes auditivos usuários do sistema de implante coclear multicanal. Sistema de multicanal Rev Bras Otorrinolaringol [online]. 2003Mar;69(2):227-33

2. Fetterman BL, Domico EH. Speech recognition in background noise of cochlear implant patients. Otolaryngol Head Neck Surg. 2002Mar;126(3):257-63.

3. Won JH, Drennan WR, Rubinstein JT. Spectral-ripple resolution correlates with speech reception in noise in cochlear implant users. J Assoc Res Otolaringol. 2007Sep;8(3):384-92.

4. Margo V, Schweitzer C, Gail F. Comparisons of Spectra 22 performance with and without an additional noise reduction processor. Sem Hear. 1997;18(4):405-15.

5. Mancini P, Bosco E, D"agosta L, Traisci G, Nicastri M, Capelli G, et al. Implementation of perceptual channels in children implanted with a HiRes 90K device. Acta Otolaryngol. 2009Dec;129(12):1442-50. 27:1-9.

6. Firszt JB, Holden LK, Skinner MW, Tobey EA, Peterson A, Gaggl W et al. Recognition of speech presented at soft to loud levels by adult cochlear implant recipients of three cochlear implant systems. Ear Hear. 2004Aug;25(4):375-87.

7. Geers AE. Techniques for assessing auditory speech perception and lipreading enhancement in young deaf children. Volta $\mathrm{R}$. 1994Nov;96(5):85-96.

8. Bevilacqua MC, Delgado EMC, Moret ALM. Estudos de casos clínicos de crianças do Centro Educacional do Deficiente Auditivo
(CEDAU), do Hospital de Pesquisa e Reabilitação de Lesões LábioPalatais - USP. In: XI Encontro Internacional de Audiologia; 1996, 30 de março a 02 de abril. Anais: Bauru, Brasil. p. 187.

9. Bevilacqua MC, Banhara MR, Da Costa EA, Vignoly AB, Alvarenga KF. The Brazilian Portuguese hearing in noise test. Int J Audiol. 2008Jun;47(6):364-5.

10. Nilson M, Soli SD, Sullivan JA. Development of the hearing in noise test for the measurement of speech reception thresholds in quiet and in noise. J Acoust Soc Am. 1994Feb;95(2):1085-99.

11. Costa EA. Estudo da correlação entre audiometria tonal e o reconhecimento de monossílabos mascarados por fala competitiva nas perdas auditivas induzidas pelo ruído. Acta Awho. 1995JanAbr;14(1):27-35.

12. Sarant JZ, Blamey PJ, Dowell RC, Clark GM, Gibson WPR. Variation in speech perception scores among children with cochlear implants. Ear Hear. 2001Feb;22(1): 18-28

13. Moret ALM. Implante coclear: audição e linguagem em crianças deficientes auditivas neurossensoriais profundas pré-linguais [tese]. Bauru: Hospital de Reabilitação de Anomalias Craniofaciais, Universidade de São Paulo; 2002.

14. Müller-Deile J, Kortmann T, Hoppe U, Hessel H, Morsnowski A. Improving speech comprehension using a new cochlear implant speech processor. HNO. 2009; 57(6):567-74.

15. Cochlear Limited. Nucleus ${ }^{\circledR}$ Freedom ${ }^{\mathrm{TM}}$ for previous generation implants. Implantable Hearing Solutions. 2007;1:7-11.

16. Santarelli R, Magnavita V, Filippi RD, Ventura L, Genovese E, Arslan E. Comparison of speech perception performance between Sprint/Esprit 3G and Freedom processors in children implanted with nucleus cochlear implants. Otol Neurotol. 2009Apr;30(3):304-12.

17. McConkey Robbins A, Koch DB, Osberger MJ, ZimmermanPhillips S, Kishon-Rabin L. Effect of age at cochlear implantation on auditory skill development in infants and toddlers. Arch Otolaryngol Head Neck Surg. 2004May;130(5):570-4.

18. Dawson PW, Decker JA, Psarros CE. Optimizing dynamic range in children using the nucleus cochlear implant. Ear Hear. 2004Jun;25(3):230-41.

19. Pjil S, Clark M, Hazzard L, Menapace CM. North American Freedom ${ }^{\mathrm{TM}}$ clinical trial: preliminary results in adults. Cochlear Ltd: white paper, 2006 